\title{
Surviving strangulation: evaluation of non-fatal strangulation in patients presenting to a tertiary care sexual assault and partner abuse care program
}

\author{
Zachary MacDonald $^{1} \cdot$ Debra Eagles $^{1,2,3} \cdot$ Krishan Yadav $^{1,3} \cdot$ Katherine Muldoon $^{3} \cdot$ Kari Sampsel $^{1,3}$
}

Received: 13 November 2020 / Accepted: 7 July 2021 / Published online: 17 August 2021

(C) The Author(s), under exclusive licence to Canadian Association of Emergency Physicians (CAEP)/ Association Canadienne de Médecine d'Urgence (ACMU) 2021

\begin{abstract}
Background Non-fatal strangulation is a dangerous mechanism of injury among survivors of intimate partner violence and sexual assault, with inadequate evidence to guide investigation in the emergency department (ED). The primary objective is to identify the proportion of intimate partner violence and sexual assault where non-fatal strangulation occurs, and to describe the sequelae of injuries.

Methods Health records review of patients treated at the Sexual Assault and Partner Abuse Care Program (SAPACP) and/ or Trauma Program at a tertiary level hospital between January 2015 and December 2018. Eligible patients were greater than 16 years old, seen by the SAPACP or trauma team for intimate partner violence and sexual assault, and had a non-fatal strangulation injury. Data were abstracted from the standardized assessment completed by the SAPACP nurse. Descriptive statistics were used.

Results We identified 209 eligible cases of non-fatal strangulation, among 1791 patient presentations to the SAPACP. Median patient age was 27 years, and $97.6 \%$ were female. Computed tomography (CT) of the head was obtained in $22.5 \%$, and CT angiography (CTA) of the head and neck in $6.2 \%$ of cases. Eleven significant injuries were identified. Two cases of vascular abnormalities: internal carotid artery indentation with possible intramural hematoma and possible internal carotid artery dissection. Other injuries included delayed bilateral subdural hematomas, a depressed skull fracture, and six nasal fractures. Conclusion We found over 10\% prevalence of non-fatal strangulation in survivors of intimate partner violence and sexual assault. There was a low rate of clinically important injury on the index ED visit secondary to non-fatal strangulation. Severe injury was primarily secondary to concomitant trauma, and utilization of CTA in this cohort was low. Increased awareness is needed among ED physicians regarding the need to consider CTA head and neck.
\end{abstract}

Keywords Trauma $\cdot$ Intimate partner violence $\cdot$ Sexual assault $\cdot$ Strangulation

\section{Résumé}

Contexte La strangulation non mortelle est un mécanisme dangereux de blessures chez les survivants de violence conjugale et d'agression sexuelle, avec des preuves insuffisantes pour guider l'enquête aux services d'urgence (SU). L'objectif principal est d'identifier la proportion de violence conjugale et d'agression sexuelle où survient un étranglement non mortel, et de décrire les séquelles des blessures.

Méthodes Examen des dossiers médicaux des patients traités au programme de soins pour les agressions sexuelles et les abus de partenaires (SAPACP) et/ou au programme de traumatologie d'un hôpital de niveau tertiaire entre janvier 2015 et décembre 2018. Les patients éligibles avaient plus de 16 ans, étaient vus par le SAPACP ou l'équipe de traumatologie pour

Kari Sampsel

ksampsel@toh.ca

1 Department of Emergency Medicine, University of Ottawa, The Ottawa Hospital-Civic Campus, Ottawa, ON K1Y 4E9, Canada

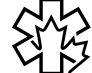

2 School of Epidemiology and Public Health, University of Ottawa, Ottawa, ON, Canada

Ottawa Hospital Research Institute, Ottawa, ON, Canada 
des violences entre partenaires intimes et des agressions sexuelles, et présentaient une blessure non mortelle par strangulation. Les données ont été extraites de l'évaluation standardisée réalisée par l'infirmière du SAPACP. Des statistiques descriptives ont été utilisées.

Résultats Nous avons identifié 209 cas éligibles de strangulation non fatale, parmi les 1791 présentations de patients au SAPACP. L'âge médian des patients était de 27 ans, et 97,6 \% étaient des femmes. La tomodensitométrie (TDM) de la tête était réalisée dans $22,5 \%$ des cas, et l'angiographie TDM de la tête et du cou dans 6,2\% des cas. Onze blessures significatives ont été identifiées. Deux cas d'anomalies vasculaires : indentation de l'artère carotide interne avec possible hématome intramural et possible dissection de l'artère carotide interne. Les autres blessures comprenaient des hématomes sous-duraux bilatéraux retardés, une fracture du crâne déprimée et six fractures nasales.

Conclusion Nous avons constaté une prévalence de plus de $10 \%$ de strangulation non mortelle chez les survivants de violence conjugale et d'agression sexuelle. Il y avait un faible taux de blessures cliniquement importantes lors de la visite aux urgences de référence, secondaires à une strangulation non fatale. Les lésions graves étaient principalement secondaires à des traumatismes concomitants, et le recours à l'angiographie dans cette cohorte était faible. Il est nécessaire de sensibiliser davantage les médecins des urgences à la nécessité d'envisager une angioplastie de la tête et du cou.

\section{Introduction}

\section{Clinician Capsule \\ What is known about the topic?}

Non-fatal strangulation is a dangerous mechanism of injury, and there are many reported cases of significant and delayed injury.

What did this study ask?

What is the prevalence of non-fatal strangulation among survivors of intimate partner violence and sexual assault?

\section{What did this study find?}

We found greater than $10 \%$ prevalence of non-fatal strangulation, with significant injury being uncommon and primarily resulting from concomitant trauma.

Why does this study matter to clinicians?

Although optimal screening patterns remain unclear, physicians should consider computed tomography angiography in survivors of non-fatal strangulation with high-risk features.

Non-fatal strangulation is a dangerous mechanism of injury among populations affected by intimate partner violence and sexual assault. The Ottawa Hospital (TOH) implemented a strangulation protocol in 2016 for patients presenting to the Sexual Assault and Partner Abuse Care Program (SAPACP). This protocol is based off the broad guidelines created by the International Association of Forensic Nursing and adapted to our local best practice patterns [1].

The primary objective was to identify the proportion of intimate partner violence and sexual assault where nonfatal strangulation occurs, and to describe the medical sequelae of non-fatal strangulation injuries. A secondary objective was to investigate the utility of a locally adopted protocol.

\section{Methods}

We conducted a health records review from January 1st, 2015 to December 31st, 2018 of patients treated at the SAPACP of TOH. TOH is a tertiary level hospital in Ottawa, Canada. TOH SAPACP is the sole hospital-based center for intimate partner violence and sexual assault cases in the region. Ethical approval was obtained from the Ottawa Health Science Network Research Ethics Board.

Eligible patients were those presenting to the emergency department (ED), 16 years or older and seen by the SAPACP with non-fatal strangulation. Patients seen by the SAPACP nurse had a standardized assessment completed. Patients with strangulation injury were identified through a pre-constructed database that included all patients assessed by the SAPACP. Additional patient data were abstracted from $\mathrm{TOH}$ electronic medical record (EMR). Our EMR search was limited to our tertiary center. Information not found within the EMR was assumed absent.

Data abstraction was performed by a single un-blinded abstractor. Each chart was searched for any repeat encounter occurring 30 days after the initial encounter to assess for missed injury. Patients with no repeat encounter were assumed lost to follow-up.

The primary outcome was the proportion of intimate partner violence and sexual assault cases where non-fatal strangulation occurred, and the clinical sequelae of non-fatal strangulation. Secondary outcome measures were provider adherence to non-fatal strangulation protocol, and the impact of protocol implementation on rate and yield of diagnostic imaging.

Simple descriptive statistics were used. Medians with interquartile range were used to describe non-normally distributed continuous variables, and categorical variables were described using percentages. Soft-tissue injury 
was sub-classified as mild (no intervention), moderate (required intervention or surgical consultation), or severe (required surgery or hospitalization).

The criteria from the strangulation protocol (Table S6) were applied to each case.

Adherence to protocol was defined as a patient who fulfilled one or more of the clinical criteria for imaging and underwent computed tomography angiography (CTA) of the head and neck.

\section{Results}

Between January 2015 and December 2018, 1791 patients presented to the SAPACP program from which 209 $(11.7 \%)$ non-fatal strangulation cases were identified. Median age was 27 years (IQR: 21-36), and $97.6 \%$ were female. Manual strangulation was the primary manner of strangulation (95.7\%). The SAPACP program had previously assessed $10.5 \%$ of patients for prior cases of intimate partner violence and sexual assault.

On assessment, 25 (12.0\%) patients reported loss of consciousness. Symptoms reported included headache (41.6\%), dizziness (39.2\%), and change in voice and/or hoarseness (23.9\%). On examination, 52.6\% reported neck tenderness. Facial edema, ecchymosis, and erythema was common for both the head (30.1\%), and neck (40.7\%).

CT head was common with 47 (22.5) patients undergoing this investigation. A small proportion of patients (6.2\%) underwent CTA of the head and neck as part of their diagnostic evaluation.

Mild soft-tissue injury was common (74.2\%). Two patients had moderate injuries: a facial laceration requiring repair, and peri-orbital hematoma requiring outpatient ophthalmology follow-up. Significant injuries are documented within Table 1 and are further outlined in detail in the accompanied appendix (Table S5). Two cases of vascular abnormalities were identified: internal carotid artery indentation with possible intramural hematoma and a possible internal carotid artery dissection versus carotid web. For two additional patients, other important injuries included subdural hematomas on repeat imaging and a depressed skull fracture. One patient had subcutaneous emphysema secondary to oropharyngeal perforation.

Prior to protocol implementation, only one patient underwent CTA. Following protocol implementation imaging was recommended in 46/108 (42.6\%) patients. Protocol adherence was poor with only $23.9 \%$ of patients undergoing CTA as recommended. There were a total of 13 CTAs performed among the entire cohort.

\section{Discussion}

This work provides insight into the medical and radiological evaluation of non-fatal strangulation survivors. Many of the severe cases were related to the presence of concomitant head trauma, and only three cases had significant findings directly related to the strangulation event. There were two patients with vascular abnormalities: one indentation with possible intramural hematoma, one suspected carotid web versus dissection, and naso-oropharyngeal mucosa perforation.

Previous work suggests that patients may present with absence of physical exam findings [2-4] and are also at risk for delayed injury [5-8]. This makes assessment challenging and has led to increased reliance on imaging. Recent studies found a 1-2\% incidence of clinically important injuries on CTA $[9,10]$. Further study is needed to develop optimal imaging protocols. We also found few clinically significant injuries directly related to strangulation or secondary to delayed presentation.

The primary strength of our study is that all patients were assessed by an SAPACP nurse, and had standardized assessments completed.

This work had several limitations. Our data abstraction was performed by a single abstractor not blinded to the results of radiological evaluation. We emphasize that our outcome of clinically serious injuries is a hard outcome based on the results of radiological tests making it less open to subjectivity. Our confidence in our low rate of missed injury is predicated on our health records review. During repeat clinical encounters, not all patients underwent repeat neurological assessments, and $11 \%$ of patients did not have a return encounter documented. This opens the possibility for missed injury. However, it is unlikely any serious injuries were missed, as our center is the regional referral center for all neurovascular emergencies.

Finally, this was a retrospective study. It is possible that physicians and nurses did not report all signs and symptoms, and there may be an underrepresentation of findings. We are also not able to ascertain why physicians opted to not follow protocol, and what clinical factors guided physician choice to pursue imaging.

Utilization of CTA was uncommon among this cohort, as only 13 CTAs in total were performed. Adherence to proposed protocol imaging recommendations were low, and reasons for poor adherence are unknown. This makes it difficult to draw any firm conclusions about the utility of this protocol as a screening modality. Many patients presented with concomitant trauma, and diagnostic investigations may have been tailored toward other distracting injuries. ED physicians should be cognizant that 
Table 1 Diagnostic imaging, outcomes, and intervention in survivors of non-fatal strangulation $(N=209)$

\begin{tabular}{lc}
\hline Variable & Patients $N=2$ \\
\hline Diagnostic imaging, $n(\%)$ & \\
X-ray & $25(12.0)$ \\
Chest & $25(12.0)$ \\
Extremity & $11(5.3)$ \\
Facial bones/panorex & $8(3.8)$ \\
Cervical spine & $8(3.8)$ \\
Neck soft tissue & $7(3.3)$ \\
Rib/thoracic & $4(1.9)$ \\
Thoracic spine & $3(1.4)$ \\
Pelvis & $4(1.9)$ \\
Other & \\
Computed tomography & $47(22.5)$ \\
Head & $13(6.2)$ \\
Computed tomography angiography head and neck & $10(4.8)$ \\
Neck soft tissue & $2(1.0)$ \\
Cervical spine &
\end{tabular}

Injury identified on physical exam and imaging, $n(\%)$

Soft-tissue injury*

Mild

$155(74.2)$

Moderate

Severe

$1(0.5)$

Fracture

$10(4.8)$

Nasal bone

$6(2.9)$

Depressed skull

$1(0.5)$

Orbital floor

$1(0.5)$

Rib

$1(0.5)$

$2(1.0)$

$1(0.5)$

ICA indentation with possible intramural hematoma

$1(0.5)$

Carotid web versus dissection

Subdural hematoma

Oropharyngeal mucosal perforation

Specialist consultation, $n(\%)$

Social work

Psychiatry

$3(1.4)$

Otolaryngology

$3(1.4)$

Ophthalmology

$1(0.5)$

rauma

$1(0.5)$

$1(0.5)$

Neurosurgery

$1(0.5)$

$1(0.5)$

$2(1.0)$

Hospitalization, $n(\%)$

$186(89.0)$

Follow-up encounter, $n(\%) * *$

ICA-Internal Carotid Artery, *Mild—presence of pain or visible injury, Moderate-requiring intervention or surgical consultation, Severe-requiring surgery or hospitalization, **Follow-up encounter with SAPACP, or any alternative encounter within electronic medical record 
CTA is an appropriate investigation in many cases of strangulation.

\section{Conclusion}

We found over $10 \%$ prevalence of non-fatal strangulation in survivors of intimate partner violence or sexual assault. There was a low rate of clinically important injury on the index ED visit secondary to non-fatal strangulation. Severe injury was primarily secondary to concomitant trauma. Adherence to an established imaging protocol was poor, and the utilization of CTA head and neck in this cohort was low. Increased awareness is needed among ED physicians regarding the need to consider CTA head and neck in survivors of non-fatal strangulation with high-risk features.

Supplementary Information The online version contains supplementary material available at https://doi.org/10.1007/s43678-021-00176-x.

Author contribution The author contributions were as follows: ZM was responsible for study coordination, data abstraction and analysis, and writing of the manuscript. KS and KM conceived the idea, coordinated the study, and contributed to manuscript revisions. KY and DE provided considerable assistance with study design, data analysis, and manuscript revisions. ZM had full access to all of the data in the study and takes responsibility for the integrity of the data and the accuracy of the data analysis.

Funding None.

\section{Declarations}

Conflict of interest None to declare.

\section{References}

1. The Evaluation and Treatment of Non-Fatal Strangulation in the Health Care Setting. International Association of Forensic Nurses. 2016. https://cdn.ymaws.com/www.forensicnurses.org/ resource/resmgr/position_papers/Strangulation_Position_Paper. pdf. Accessed 2 April 2021.

2. Holbrook DS, Jackson MC. Use of an alternative light source to assess strangulation victims. J Forensic Nurs. 2013;9(3):140-5.

3. Shields LB, Corey TS, Weakley-Jones B, Stewart D. Living victims of strangulation: a 10-year review of cases in a metropolitan community. Am J Forensic Med Pathol. 2010;31(4):320-5.

4. Strack GB, McClane GE, Hawley D. A review of 300 attempted strangulation cases. Part I: criminal legal issues. J Emerg Med. 2001;21(3):303-9.

5. Clarot F, Vaz E, Papin F, Proust B. Fatal and non-fatal bilateral delayed carotid artery dissection after manual strangulation. Forensic Sci Int. 2005;149(2-3):143-50.

6. Di Paolo M, Guidi B, Bruschini L, Vessio G, Domenici R, Ambrosino N. Unexpected delayed death after manual strangulation: need for careful examination in the emergency room. Monaldi Arch Chest Dis. 2009;71(3):132-4.

7. McClane GE, Strack GB, Hawley D. A review of 300 attempted strangulation cases Part II: clinical evaluation of the surviving victim. J Emerg Med. 2001;21(3):311-5.

8. Sethi PK, Sethi NK, Torgovnick J, Arsura E. Delayed left anterior and middle cerebral artery hemorrhagic infarctions after attempted strangulation: a case report. Am J Forensic Med Pathol. 2012;33(1):105-6.

9. Matusz EC, Schaffer JT, Bachmeier BA, et al. Evaluation of nonfatal strangulation in alert adults. Ann Emerg Med. 2020;75(3):329-38.

10. Zuberi OS, Dixon T, Richardson A, Gandhe A, Hadi M, Joshi J. $\mathrm{CT}$ angiograms of the neck in strangulation victims: incidence of positive findings at a level one trauma center over a 7-year period. Emerg Radiol. 2019;26(5):485-92. 